\title{
Enfermedad multianeurismática: a propósito de un caso
}

\author{
Isabel María Manosalbas-Rubio, Esther Doiz-Artázcoz, \\ Ana Margarita Ruales-Romero y Manuel Rodríguez-Piñero
}

\section{Multi-aneurysmal disease: A case report}

Introduction: Multiple artery aneurysms are a rare pathological condition which may be caused by different etiologies. Therefore, its location, morphology and clinical presentation may vary in a case to case basis. Case report: A 51-year-old woman, prior history of dyslipedemia presents with upper abdominal pain. Abdominal tomographic scans showed aneurysm of the pancreaticduodenal artery and retroperitoneal hematoma. Emergent surgical evacuation of the hematoma was performed, with no other findings. In the postoperative period, the patient suffers hypertensive crisis and a new tomographic scan is conducted observing multiple dilations in different visceral arteries. The patient is treated conservatively and is being studied for a possible vasculitis. Discussion: Multi-aneurysmatic artery disease is a very rare entity, its etiology is determined by clinical and histopathological correlation. Although establishing a diagnosis in which the clinical presentation completely corresponds, is a real challenge. Unlike degenerative aneurysms due to atherosclerosis, multi-aneurysmatic disease commonly involves visceral arteries. Open surgery is considered safe treatment option and should be established in the segments causing symptoms. Endovascular treatment is less invasive, being the technique of choice in patients with high comorbidity and in cases of complicated surgery with rupture.

Key words: multiple artery aneurysms; multi-aneurysmatic disease; vasculitis.

\section{Resumen}

Introducción: La enfermedad multianeurismática es una entidad patológica poco conocida, la cual presenta diversas etiologías, por lo que su localización, morfología y formas de presentación varían de un caso a otro. Caso clínico: Mujer de 51 años dislipémica. Acude por epigastralgia. Se realiza TC abdominal con resultados de hematoma retroperitoneal y aneurisma en arteria pancreática duodenal. Se practica cirugía urgente para evacuación del hematoma sin encontrarse más hallazgos. Durante el ingreso presenta crisis hipertensivas y se realiza nuevo AngioTC donde se visualizan múltiples lesiones en distintas arterias viscerales con posibilidad diagnóstica de vasculitis. Se decide no intervención en el momento actual y estudio de filiación. Discusión: La enfermedad multianeurismática es una entidad poco común, cuya etiología se determina de acuerdo con su correlación clínica e histopatológica con diversas posibles causas; sin embargo, establecer un diagnóstico en donde el cuadro clínico coincida al 100\%, es un desafío. Es muy frecuente la afectación de las arterias viscerales a diferencia de los aneurismas de origen ateroesclerótico. El tratamiento quirúrgico es seguro y deberá iniciarse en los segmentos que estén causando la sintomatología. El tratamiento endovascular es menos invasivo siendo la técnica de elección en pacientes con elevada comorbilidad y en los casos de cirugía complicada con rotura.

Palabras clave: múltiples aneurismas; enfermedad multianeurismática; vasculitis.
Angiología y Cirugía Vascular. Hospital Universitario Puerta del Mar. Cádiz.

Recibido el 12 de julio y aceptado para publicación el 27 de septiembre de 2018.

Correspondencia a: Dra. Isabel María Manosalbas-Rubio isamaru91@gmail.com 


\section{CASO CLÍNICO}

\section{Introducción}

La enfermedad multianeurismática es muy poco conocida, con sólo 178 casos en la literatura y sin ningún dato de incidencia. La edad a la que se diagnostica varía ampliamente desde el estado fetal $^{1}$ hasta la juventud o edad adulta. La morfología más frecuente es la sacular, independientemente de la localización o la etiología y la incidencia en hombres y mujeres no presenta diferencias significativas, sin asociación o historia familiar presente. Fisiopatológicamente se produce una respuesta anormal de las células musculares lisas y neutrófilos, produciendo más elastasa intracelular y conduciendo a degeneración aneurismática de la pared arterial. Pueden ocurrir asociados con desórdenes del tejido
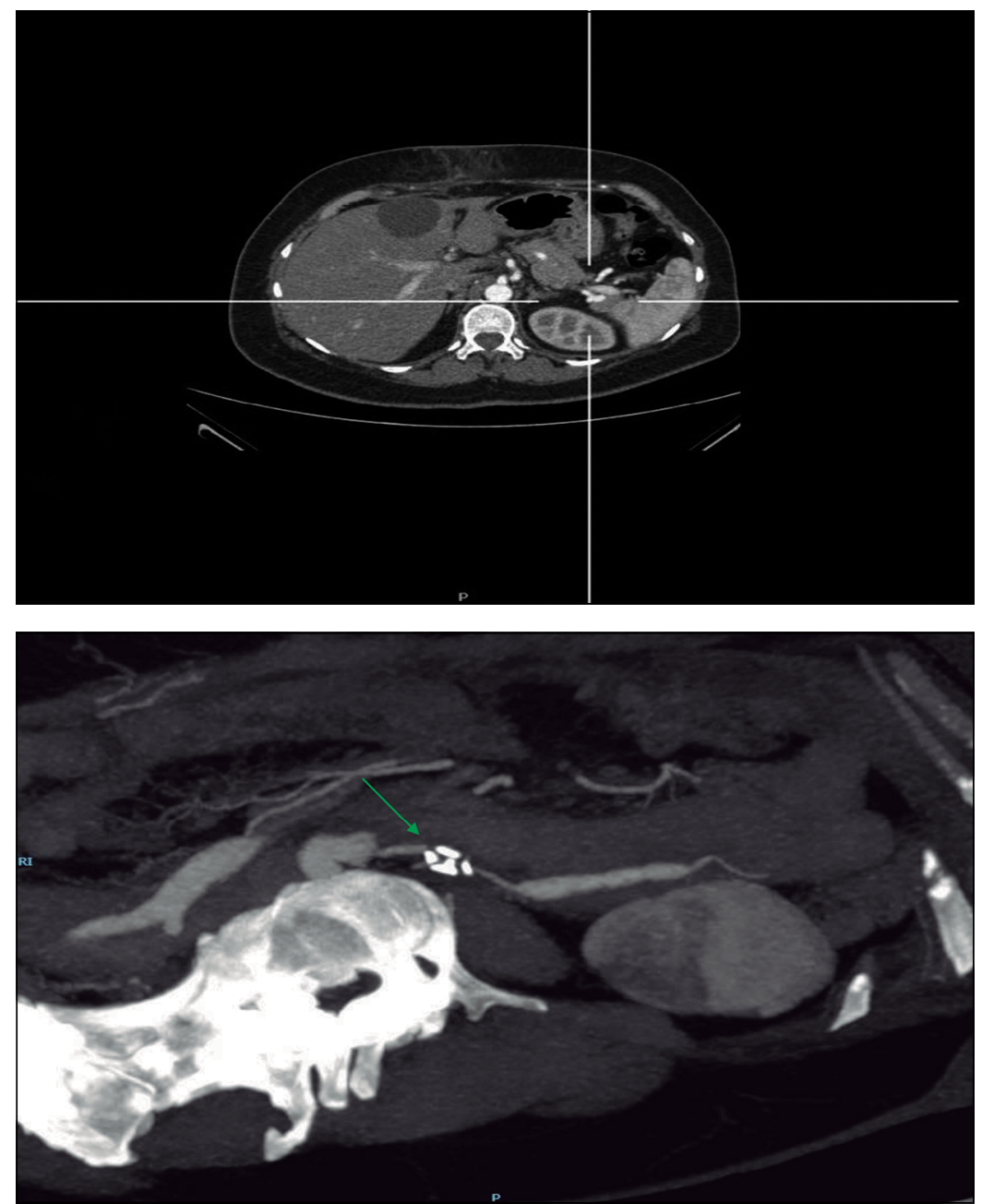

Figuras 1 y 2. En el segmento distal de la arteria esplénica se identifica una pequeña dilatación a nivel posterior, sugestiva de pequeño aneurisma. conectivo como el síndrome de Ehlers-Danlos y el síndrome de Marfan. También pueden ocurrir en asociación con varios tipos de arteritis $^{2}$ como poliarteritis nodosa ${ }^{3}$, enfermedad de Takayasu ${ }^{4}$, enfermedad de Kawasaki o el síndrome de Loeys-Dietz ${ }^{5}$. Su presentación es variada aunque lo más frecuente es la afectación de extremidades superiores (92\%), región aorto-ilíaca $(92 \%)$ y vasos renales y mesentéricos $(77 \%)$.

\section{Caso clínico}

Mujer de 51 años con antecedente médico de dislipemia en tratamiento y quirúrgico de histerectomía. Acude al Servicio de Urgencia por dolor abdominal en epigastrio irradiado en cinturón, junto a náuseas y vómitos de unas doce horas de evolución. Afebril y normotensa. A la palpación abdominal, dolor en hipogastrio derecho sin signos de irritación peritoneal. Hemograma con discreta leucocitosis y resto de exámenes dentro de la normalidad. Ante la sospecha clínica de colecistitis se le realiza una ecografía abdominal que es anodina, por lo que es dada de alta para control y evolución domiciliaria.

Acude de nuevo a urgencias $48 \mathrm{~h}$ después por empeoramiento de la sintomatología. Se le realiza una TC abdominal donde se observa hematoma retroperitoneal y pequeño aneurisma que parece localizado en la arteria pancreático-duodenal el cual puede ser el origen del sangrado. Ante estos hallazgos consulta con Servicio de Cirugía General quienes realizan de urgencia laparotomía exploradora y evacuación del hematoma sin visualización de otros hallazgos. La paciente permanece $24 \mathrm{~h}$ en la Unidad de Recuperación Postanestésica y sube a la planta de hospitalización donde sufre crisis hipertensivas que son estudiadas por Medicina Interna. Se le realiza un doppler renal donde se objetiva la presencia de un angiomiolipoma izquierdo sin otras alteraciones. Se le realiza un estudio de catecolaminas que no presenta hallazgos patológicos y estudio de inmunidad negativo.

La paciente evoluciona favorablemente y se realiza Angio-TC de aorta abdominal de control en el que se objetiva la presencia de una morfología característica del tronco celíaco que podría estar en relación con síndrome del ligamento arcuato, un pequeño aneurisma a nivel posterior en el segmento distal de la arteria esplénica (Figuras 1 y 2), disminución del calibre generalizado de la arteria hepática común de aspecto filiforme con malformación vascular en el lóbulo hepático, pequeño aneurisma sacular de la arteria pancreaticoduodenal (Figura 3) 
y dilatación aneurismática, de aspecto arrosariado, de un segmento de unos $49 \mathrm{~mm}$ de una rama de la arteria mesentérica inferior (Figura 4).

Con la sospecha diagnóstica de múltiples dilataciones aneurismáticas de ramas de las arterias abdominales consultan con el Servicio de Cirugía Vascular, quienes decidimos tratamiento conservador dado las pequeñas dimensiones de los aneurismas en el momento actual. La paciente es dada de alta para seguimiento en consulta externa y remitida a Medicina Interna para estudio causal y de filiación.

\section{Discusión}

La enfermedad multianeurismática es una entidad clínica poco frecuente, y cuya etiología, localización, morfología y forma de presentación varían de un caso a otro. Suponen un reto para el profesional sanitario, tanto en el diagnóstico como en el tratamiento ${ }^{5}$.

La casuística encontrada en la literatura es muy reducida no llegando a los doscientos casos por lo que su diagnóstico y seguimiento supone un reto para el equipo quirúrgico debiendo de individualizar en cada caso y según etiología y clínica. Aparece asociada, generalmente, a síndrome de Marfan, síndrome de Loeys-Dietz ${ }^{5}$, Ehlers Danlos ${ }^{6}$, Takayasu o Poliarteritis Nodosa ${ }^{7}$, entre otras. Los pacientes suelen presentar sintomatología variada de acuerdo con la localización de los aneurismas, la cual suele diagnosticarse de manera casual.

La enfermedad multianeurismática es una patología de difícil diagnóstico y tardío tratamiento. Suelen ser asintomáticos hasta que debutan con signos de complicación como rotura o clínica de compresión local. La edad del diagnóstico es muy variada desde la edad fetal hasta la juventud o edad adulta. En los estadios iniciales el hallazgo suele ser en forma de aneurisma aislados mientras que de forma tardía el debut suele ser de forma múltiple.

La TC es la prueba diagnóstica de elección ${ }^{8}$ si bien, la presencia de una masa abdominal pulsátil mediante ecodoppler, debe hacernos sospechar dicha patología. En la enfermedad multianeurismática es muy frecuente la afectación de las arterias viscerales, a diferencia de los aneurismas de origen ateroesclerótico, en donde aproximadamente el $5 \%$ de las arterias viscerales se encuentran comprometidas, de estas, sólo el 5\% afecta al tronco celíaco y la arteria más frecuentemente afectada es la arteria esplénica, seguida de la arteria renal y la arteria hepática. Del 10\% al 20\% de los aneurismas viscerales se rompen espontáneamente con una mortalidad estimada entre el $20 \%$ y el $70 \%$. En los pacientes con enfermedad multianeurismática se han detectado aneurismas viscerales gigantes totalmente asintomáticos e íntegros.

El tratamiento conservador y el control mediante técnicas de imagen es el indicado en la mayoría de los aneurismas de arterias viscerales. La falta de estudios prospectivos determina que no hay un consenso estandarizado en las indicaciones de tratamiento de los aneurismas viscerales. De las series de casos sometidos a seguimiento y a tratamiento se derivan las indicaciones de tratamiento quirúrgico en las que están de acuerdo los diferentes auto-

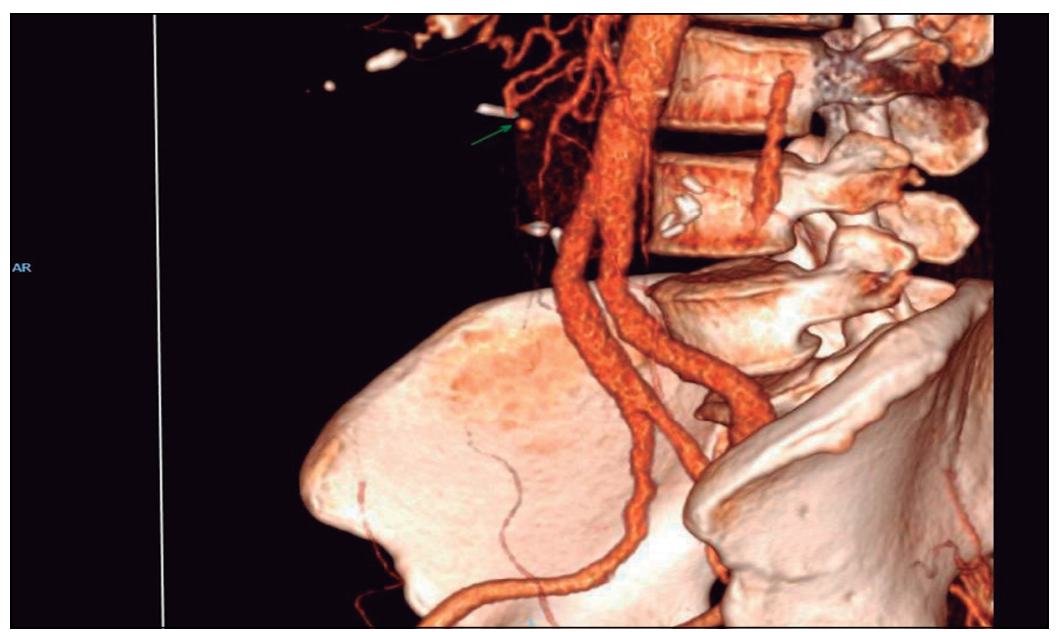

Figuras 3. Se identifica una imagen nodular hiperdensa a nivel de la cabeza pancreática, de unos $6 \mathrm{~mm}$, que parece depender de una rama distal de la arteria mesentérica superior (posiblemente de la arteria pancreático-duodenal) que sugiere un pequeño aneurisma sacular.

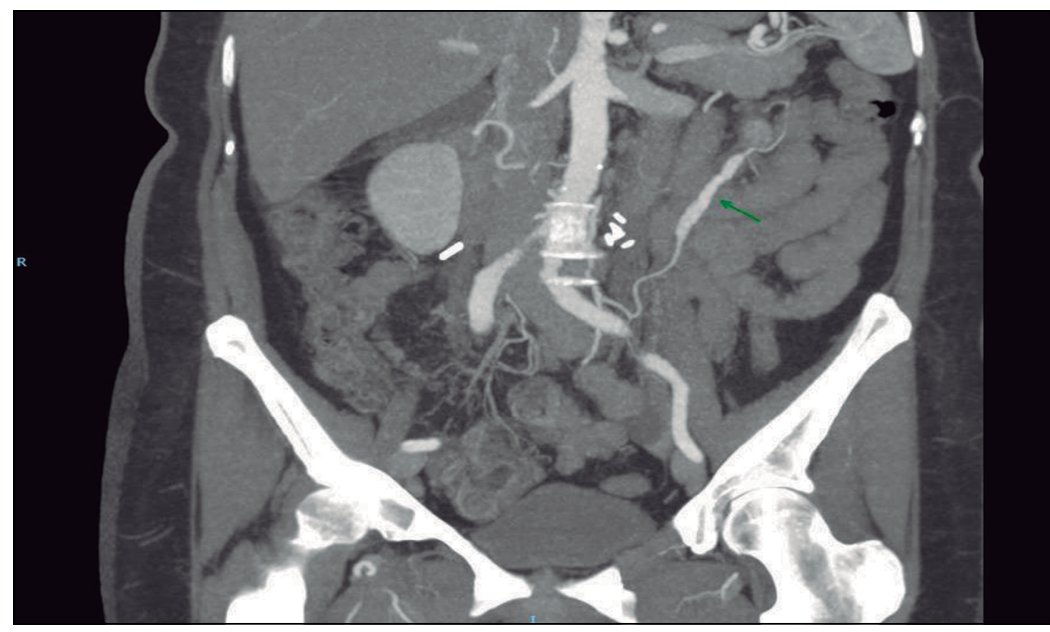

Figuras 4. Dilatación aneurismática, de aspecto arrosariado, de un segmento de unos $49 \mathrm{~mm}$ de una rama de la arteria mesentérica inferior. 
res. El tratamiento debe de realizarse en todos los pseudoaneurismas, los aneurismas sintomáticos o rotos, aquellos mayores de $20 \mathrm{~mm}$ o con un rápido crecimiento ( $>5 \mathrm{~mm}$ anual) y aquellos de menor tamaño que se diagnostiquen en mujeres gestantes o en edad fértil.

El objetivo del tratamiento ${ }^{2,8}$, tanto quirúrgico como endovascular, consiste en excluir el saco aneurismático de la circulación preservando el flujo a los órganos distales al mismo. Independientemente del tratamiento escogido hemos de tener presente la localización del aneurisma y la arteria afectada con el fin de preservar la colateralidad del vaso y órgano afecto.

El tratamiento quirúrgico clásico es seguro y consiste en el abordaje abierto para exclusión del aneurisma, asociando en lo posible restablecer la continuidad vascular. La cirugía ofrece las ventajas de una mayor durabilidad y menor necesidad de seguimiento, además, de una mortalidad teórica inferior al $0,5 \%$ en la cirugía programada ${ }^{2,8}$.

El tratamiento mediante técnicas endovasculares ha presentado una evolución al alza considerándola de elección en muchos casos. Sus ventajas radican en que es menos invasivo, permite reducir la estancia hospitalaria y mantener una buena calidad de vida en el posoperatorio inmediato. Es la opción de elección para pacientes con elevada comorbilidad y cada vez más en los casos de cirugía complicada con rotura $^{8}$. La rotura de un aneurisma visceral supone una urgencia quirúrgica con una elevada morbimortalidad.

\section{Responsabilidades éticas}

Protección de personas y animales. Los autores declaran que para esta investigación no se han realizado experimentos en seres humanos ni en animales.

Confidencialidad de los datos. Los autores declaran que han seguido los protocolos de su centro de trabajo sobre la publicación de datos de pacientes.

Derecho a la privacidad y consentimiento informado. Los autores declaran que en este artículo no aparecen datos de pacientes.

Conflictos de interés: No hay.

\section{Referencias}

1. Halpern V, O`Coonor J, Murello M, Siegel D, R. Cohen J. Multiple idiopathic arterial aneurysms in children: a case report and review of literature. J Vasc Surg. 1997; 25:949-56

2. Jennette JC, Falk RJ, Andrassy K, Bacon PA, Churg J, Gross WL, et al. Nomenclature of systemic vasculitides. Proposal of an international consensus conference. Arthritis Rheum. 1994;37:187-92.
3. Guillevin L, Pagnoux C, Teixeira. Polyarteritis nodosa. In Ball GV, Bridges SL, editors: Vasculitis, ed 2, 2008, Oxford, pp 335-53.

4. Vanoli M, Daiana E, Salvarani C. Takayasu`s arteritis: a study of 104 Italian patients. Arthritis Rheum. 2005;53:100-7.

5. Hinojosa CA, Anaya-Ayala JE, LaparraEscareno H, Torres-Machorro A, Lizola R. Complex aortic and bilateral renal artery aneurysm repair in a young patient with multiple arterial aneurysm syndrome.
Journal of Vascular Surgery Cases and Innovative Techniques 2016;2:84-7.

6. Dieter R, Murtaugh T, Black J. Coronary arteriomegaly in a patient with Ehlers Danlos Syndrome and multiple aneurysms: a case report. Angiology 2003;54:733-6.

7. Bonsib SM. Polyarteritis nodosa. Semin Diagn Pathol. 2001;18:14-23.

8. Björck M, Koelemay M, Acosta S, Bastos-Goncalves F, Kölbel T, Kolkman JJ. Eur J Vasc Endovasc Surg. 2017;53:460e-510. 